\title{
Prediction of lymphovascular space invasion using a combination of tenascin-C, Cox-2, and PET/CT radiomics in patients with early-stage cervical squamous cell carcinoma
}

\section{Xiaoran Li}

Shengjing Hospital of China Medical University Department of Radiology

Chen Xu

Shengjing Hospital of China Medical University Department of Radiology

Yang Yu

Shengjing Hospital of China Medical University Department of Radiology

\section{Yan Guo}

GE Healthcare

Hongzan Sun ( $\square$ sunhongzan@126.com )

Shengjing Hospital of China Medical University Department of Radiology https://orcid.org/0000-00024724-5828

\section{Research article}

Keywords: PET/CT, radiomics, machine learning, lymphovascular space invasion, cervical squamous cell carcinoma

Posted Date: October 6th, 2020

DOl: https://doi.org/10.21203/rs.3.rs-75399/v1

License: (1) (i) This work is licensed under a Creative Commons Attribution 4.0 International License. Read Full License

Version of Record: A version of this preprint was published at BMC Cancer on July 28th, 2021. See the published version at https://doi.org/10.1186/s12885-021-08596-9. 


\section{Abstract \\ Background}

Lymphovascular space invasion is an independent prognostic factor in early-stage cervical cancer. However, there is a lack of non-invasive methods to detect lymphovascular space invasion. Some researchers found that Tenascin-C and Cyclooxygenase-2 was correlated with lymphovascular space invasion. Radiomics has been studied as an emerging tool for distinguishing tumor pathology stage, evaluating treatment response, and predicting prognosis.

\section{Materials and methods}

Eighty-six patients with early-stage cervical squamous cell carcinoma who underwent PET/CT examination were retrospectively analyzed. 401 radiomics features based on PET/CT images were extracted and integrated into radiomics score (Rad-score). Immunohistochemical analysis was performed to evaluate TNC and COX-2 expression. Mann-Whitney $U$ test was used to distinguish differences in the Rad-score, TNC, and COX-2 between LVSI and non-LVSI groups. The correlations of characteristics were tested by Spearman analysis. Machine learning models including radiomics model, protein model and combined model were established by logistic regression algorithm and evaluated by ROC curve. Pairwise comparisons of ROC curves were tested by DeLong test.

\section{Results}

The Rad-score of patients with LVSI was significantly higher than those without. A significant correlation was shown between LVSI and Rad-score $(r=0.631, p<0.001)$. TNC was correlated to both the Rad-score $(r$ $=0.244, p=0.024)$ and COX-2 $(r=0.227, p=0.036)$. The radiomics model had the best predictive performance among all models (AUC $=0.914, p<0.001)$ in training dataset. However, in testing dataset, the combined model had better efficiency for predicting LVSI than other models (AUCs: 0.801 vs. 0.756 and 0.801 vs. 0.631 , respectively).

\section{Conclusion}

The machine learning model of the combination of PET radiomics with COX-2 and TNC provides a new tool for detecting LVSI in patients with early-stage cervical cancer.

\section{Background}

According to current global cancer statistics, cervical cancer ranks second among female tumors, and the number of new cases in developing countries is increasing annually and involving younger women[1]. Some studies have shown that LVSI is an independent prognostic factor in early-stage cervical cancer[2- 
6]. LVSI was detected in $17.8 \%$ of early-stage cervical cancer cases in the initial pathological examination[7]. Herr et al[3] found that the presence of satellite LVSI was associated with significantly reduced overall survival and disease-free survival, compared with lack of any LVSI. According to Memarzadeh et al[4], $86 \%$ of patients with perineural involvement in the parametria had evidence of parametrial LVSI, and the multivariate analysis revealed that large tumor size $(>4 \mathrm{~cm})$, parametrial perineural invasion, cervical LVSI, and tumor depth $(>2 / 3)$ were significant simultaneous predictors of recurrence for early-stage cervical cancer $(p<0.05)$. Subsequently, Pol et al[5] also confirmed that conjoined and satellite LVSIs were significantly associated with recurrence and survival. In a retrospective cohort study, LVSI was shown to be an independent factor that affects overall survival $(p=0.009)$ and progression-free survival $(p=0.006)$ in patients with early-stage cervical cancer[6]. However, LVSI could only be confirmed by postoperative pathology.

In recent years, radiomics based on PET/CT imaging has been studied as an emerging tool for distinguishing tumor pathology stage, evaluating treatment response, and predicting prognosis[8, 9]. Lambin et al[10] proposed the radiomics hypothesis that intratumoral heterogeneity evaluated by imaging could be the expression of genomic heterogeneity, as tumors with genomic heterogeneity are more likely to metastasize.

In previous studies, some protein molecules expression have been shown to be associated with lymphangiogenesis, lymph node metastasis[11], and lymphovascular invasion in the early-stage cervical cancer. Hoellen et al[12] determined that cyclooxygenase-2 (COX-2) expression was significantly associated with LVSI $(p=0.017)$. The expression of tenascin-C (TNC) in invasive cervical carcinoma was markedly increased[13]. Pilch[13] et al's research showed that, in $84 \%$ of the cases examined, a strong TNC immunoreactivity was noted around and within the tumor cell nests. TNC is not only associated with epithelial-mesenchymal transition, proliferation, and migration of cancer cells, but it also facilitates the formation of cancer stroma, including desmoplasia and angiogenesis[14]. Thus, we aimed to explore the association of radiomics derived from ${ }^{18} \mathrm{~F}$-fluorodeoxyglucose PET/CT imaging combined with COX-2 and TNC expressions with LVSI in early cervical cancer and to establish a machine learning model of the combination of PET/CT radiomics, COX-2, and TNC to predict LVSI in patients with early-stage cancer.

\section{Materials And Methods}

\section{Patient cohort}

This retrospective study was performed at the Department of Radiology at Shengjing Hospital in Shenyang, China. Between January 2015 and December 2018, 105 female patients with cervical cancer confirmed by biopsy pathology underwent pretreatment ${ }^{18} \mathrm{~F}$-FDG PET/CT. Inclusion criteria: (1) histologically confirmed cervical cancer with stage la-Ila determined by the 2018 International Federation of Gynecology and Obstetrics (FIGO) classifications[15]; (2) absence of other malignant tumors; (3) normal serum glucose levels before undergoing PET/CT. Of these patients, 19 subjects were excluded because of previous chemoradiotherapy before the examination $(n=5)$, tumor volume $<1 \mathrm{~cm}^{3}$ leading to image data 
being unsuitable for textural feature measurement $(\mathrm{n}=10)$, and surgery performed in another hospital $(\mathrm{n}=$ 4). Finally, 86 patients ( $42 \mathrm{LVSI}$ and 44 non-LVSI) were randomly divided into two groups, including the training and testing data sets, according to a 7:3 ratio.

\section{Immunohistochemistry}

All tissue specimens of cervical carcinoma were prepared by the Department of Pathology in our hospital. Immunohistochemical staining was performed using Leica BOND MAX ${ }^{\mathrm{TM}}$ (Leica Biosystems, Shanghai, China). For immunohistochemical detection of COX-2 and TNC protein expressions, the sections were incubated with goat anti-human COX-2 (1:400 dilution) or rabbit anti-human TNC (1:400 dilution ) polyclonal primary antibodies (both from Abcam, Shanghai, China) at $4{ }^{\circ} \mathrm{C}$ overnight. After washing, the sections were incubated with species-appropriate enzyme-conjugated anti-rabbit and anti-goat secondary antibodies. Dewaxing, antigen epitope exposure, blocking, incubation with primary antibody, development of diaminobenzidine oxidation color, hematoxylin staining, and dehydration were automatically completed by the computer.

After the tissue was sliced, it was placed on Pannoramic MIDI tissue slice scanner (3DHISTECH Ltd., Budapest, Hungary), which simultaneously moved and scanned the image, forming a file that contained all the information on the tissue section. The file could be magnified 1-400 times using the Pannoramic viewer software, and the picture could be intercepted at any location. The QuantCenter (3DHISTECH Ltd.) is an analysis software that supports the Pannoramic viewer. After image scanning is completed, the DensitoQuant software in the QuantCenter automatically recognizes and sets all dark brown areas on the tissue section as strong positive, brown yellow as moderate positive, light yellow as weak positive, and blue cell nucleus as negative. Furthermore, all strong-positive, moderate-positive, weak-positive, and negative areas (in pixels); the percentage of positive areas; and the $\mathrm{H}$-score were analyzed for each tissue[16]. All cases were divided into three groups according to the extent of staining and $\mathrm{H}$-score: negative or weakpositive, moderate-positive, and strong-positive groups[11].

\section{${ }^{18}$ F-FDG PET/CT acquisition and features extraction}

The patients rested quietly for 60 min before PET/CT (Discovery PET/CT 690; GE Healthcare, Chicago, IL, USA) scanning. All CT and PET scans were acquired with free breathing for attenuation correction and image fusion. First, low-dose non-enhanced CT images were acquired with a bulb voltage of $120 \mathrm{kV}$, auto $\mathrm{mA}$ (30-210 mA; noise index, 25), and slice thickness of $3.27 \mathrm{~mm}$. Then, the PET data were acquired after CT scanning using a three-dimensional acquisition mode at a speed of $1.5 \mathrm{~min} / \mathrm{bed}$ ( $7-8$ beds in total) and a matrix size of $192 \times 192$. The time-of-flight and point-spread function techniques were also used in the reconstruction. A volume of interest (VOI) was automatically obtained on an AW4.5 workstation (GE Healthcare). The metabolic tumor volume(MTV), total lesion glycolysis(TLG), and maximum(SUV max $_{\text {, }}$, mean $\left(S U V_{\text {mean }}\right)$, and peak $\left(S U V_{\text {peak }}\right)$ standard uptake values were measured automatically inside the segmented VOI.

The VOI based on PET images was manually drawn layer by layer around the cervical squamous cell carcinomas by two nuclear medicine physicians, who performed the task independently. Both had more 
than 10 years of experience and were blinded to patients' clinical data. The radiomics features were extracted from the PET images using AK software (Artificial Intelligence Kit, GE Healthcare, Shanghai, China)[17].

\section{Calculation of Radiomics Score}

All data analyses were processed with R version 3.5.1 (The R Foundation, Vienna, Austria). Data normalization steps such as feature transformation and standardization are needed for radiomics features due to the intrinsic differences in the range, scale, and statistical distributions of these features[18]. All features were normalized with the Z-score method.

Considering that a large number of radiomics features might be highly redundant; thus, to reduce redundant data in the machine learning model, the least absolute shrinkage and selection operator (LASSO) algorithm with 10-fold cross validation was used to filter radiomics features[19]. The selected features with non-zero coefficient were then linear combined that were weighted by their respective coefficients to build a radiomics signature, here we called radiomics score (Rad-score).

\section{Establishment of Machine learning models}

In the training data set, logistic regression algorithm was used to establish the machine learning model. Three models were built separately to predict LVSI in the training and testing data sets: the radiomics model, which only included the Rad-score parameter to predict LVSI; the protein model, which included COX-2 and TNC expression to predict LVSI; and the combined model, which included all selected parameters. In the testing data set, models were tested and evaluated independently.

\section{Statistical analysis}

All statistical analyses were performed with SPSS version 25.0 (IBM Corp., Armonk, NY, USA) and R version 3.5.1. The Mann-Whitney $U$ test is used for continuous variables, and the Pearson chi-square test is used for non-continuous variables to evaluate the feature distribution of the training dataset and the testing dataset. Spearman correlation analysis of LVSI situation with immunohistochemical data and PET imaging features was performed. Mann-Whitney $U$ test was used to distinguish differences in PET features and protein expressions between patients with early-stage cervical cancer with (LVSI group) and without (non-LVSI group) in all dataset. Multivariate logistic regression analysis was used to predict LVSI in all patients.

Receiver operating characteristic (ROC) curve was used to evaluate the performance of each machine learning model. Pairwise comparison of area under ROC curves (AUC) was performed with DeLong test. All statistical tests were performed with a two-tailed $p<0.05$ considered statistically significant.

\section{Results}

\section{Baseline Characteristics of Patients}


The patients' characteristics are summarized in Table 1. A total of 86 early-stage cervical cancer patients were enrolled in this study as the whole cohort and were further distributed randomly to either the training cohort or testing cohort. The training cohort included 61 patients with a median age of 50 years (range 3374 years) and the testing cohort had 25 patients with a median age of 51 years (range 40-58 years). 30 patients had LVSI in the training dataset that was pathologically proven (30/61) and 12 patients $(12 / 25)$ in the testing dataset. There was no significant difference of all characters between the training and testing dataset (Table 1). 
Table 1

Patient characteristics

\begin{tabular}{|c|c|c|c|}
\hline Characteristic & $\begin{array}{l}\text { Training dataset }(\mathrm{N}= \\
61)\end{array}$ & $\begin{array}{l}\text { Testing dataset }(\mathrm{N}= \\
\text { 25) }\end{array}$ & $\begin{array}{l}P \\
\text { value }\end{array}$ \\
\hline Age,median(range)years & $50(33-74)$ & $51(40-58)$ & 0.970 \\
\hline \multicolumn{4}{|l|}{ FIGO stage, No. (\%) } \\
\hline la & $18(29.50)$ & $4(16.00)$ & \\
\hline $\mathrm{lb}$ & $26(42.60)$ & $10(40.00)$ & \\
\hline Ila & $17(27.90)$ & $11(44.00)$ & \\
\hline Tumor grade, No. (\%) & & & 0.085 \\
\hline Well differentiate & $14(23.00)$ & $6(24.00)$ & \\
\hline Moderately differentiate & $29(47.50)$ & $17(68.00)$ & \\
\hline Poorly differentiate & $18(29.50)$ & $2(8.00)$ & \\
\hline $\begin{array}{l}\text { Depth of cervical stromal tumor invasion, } \\
\text { No. }(\%)\end{array}$ & & & 0.605 \\
\hline$\geq 1 / 2$ & $43(70.50)$ & 19(76.00) & \\
\hline$\nabla 1 / 2$ & $18(29.50)$ & $6(24.00)$ & \\
\hline LVSI, No. (\%) & & & 0.921 \\
\hline Yes & $30(49.20)$ & $12(48.00)$ & \\
\hline No & $31(50.80)$ & $13(52.00)$ & \\
\hline COX-2 expression No. (\%) & & & 0.400 \\
\hline Negative or Weak-Positive & $28(45.90)$ & $11(44.00)$ & \\
\hline Moderate-Positive & $22(36.10)$ & $12(48.00)$ & \\
\hline Strong-Positive & $11(18.00)$ & $2(8.00)$ & \\
\hline TNC expression No. (\%) & & & 0.975 \\
\hline Negative or Weak-Positive & $40(65.60)$ & $17(68.00)$ & \\
\hline Moderate-Positive & $16(26.20)$ & $6(24.00)$ & \\
\hline Strong-Positive & $5(8.20)$ & $2(8.00)$ & \\
\hline
\end{tabular}

\section{Rad-score based on PET radiomics features}


A total of 401 features were derived from the VOI of primary tumor on PET images that included five conventional features (SUVmax, SUVmean, SUVpeak, MTV and TLG) and 396 radiomics features (42 histogram features, 345 texture features, and 9 form factor features), as shown in Fig. 1. In the training dataset, a total of 16 most informative features with non-zero coefficient (Fig. 2) were reserved in the LASSO regression analysis when lambda chosen as 0.02 (Fig. 3), which the binomial deviance was minimum in the 10 -fold cross validation. Then rad-score was calculated using following formula:

Rad-score $(\mathrm{PET})=-0.328 *$ Correlation_angle45_offset7-0.361*InverseDifferenceMoment_angle45_offset4 + 0.291*HighIntensityLargeAreaEmphasis-0.273*LowIntensityEmphasis-

0.531*HaralickCorrelation_AllDirection_offset1_SD-

0.47*InverseDifferenceMoment_AllDirection_offset7_SD +

0.499*HighGreyLevelRunEmphasis_AllDirection_offset4_SD-

0.335*ShortRunEmphasis_AllDirection_offset7_SD-

0.482*LongRunHighGreyLevelEmphasis_AllDirection_offset1_SD + 0.415*Quantile0.025-

$0.626 *$ ClusterProminence_angle45_offset7 + 0.065*LongRunEmphasis_angle0_offset1 +

0.63*Inertia_angle45_offset4 + 0.316*InverseDifferenceMoment_angle90_offset7 +

0.507*ShortRunLowGreyLevelEmphasis_AllDirection_offset7_SD+-0.004*GLCMEnergy_AllDirection_offset7 $-0.075$

\section{Correlation of LVSI with PET features and molecular protein expressions}

The Spearman correlation analysis revealed a significant correlation between LVSI and the Rad-score $(r=$ $0.631, p<0.001)$ (Fig. 4). LVSI was also correlated to COX-2 and TNC expression $(r=0.276, p=0.01$, and $r=$ $0.333, p=0.002$, respectively). The other PET conventional parameters did not have significant correlation with LVSI or protein expressions. TNC was correlated to both the Rad-score $(r=0.244, p=0.024)$ and COX-2 $(r=0.227, p=0.036)$.

\section{Univariate analyses of the PET features and molecular protein expression with LVSI}

The results of the Mann-Whitney $U$ test are summarized in Table 2. The Rad-score, COX-2, and TNC significantly differed between the LVSI and non-LVSI groups $(p<0.005)$. The LVSI group had a higher Radscore than the non-LVSI group ( $p<0.001$, Mann-Whitney U test) (Fig. 5). 
Table 2

Differences between the LVSI and non-LVSI groups in all dataset

\begin{tabular}{|lllllll|}
\hline Variable & COX-2 & TNC & SUV $_{\text {max }}$ & TLG & SUV $_{\text {peak }}$ & Rad-score (PET) \\
\hline Mann-Whitney U & 594.000 & 517.000 & 877.500 & 896.000 & 894.500 & 251.000 \\
\hline Wilcoxon W & 1584.000 & 1507.000 & 1867.500 & 1886.00 & 1884.500 & 1241.000 \\
\hline Z & -3.096 & -3.886 & -0.402 & -0.242 & -0.255 & -5.814 \\
\hline p & 0.002 & $<0.001$ & 0.688 & 0.809 & 0.799 & $<0.001$ \\
\hline
\end{tabular}

Mann-Whitney U test was used to distinguish differences between the LVSI and non-LVSI groups.

COX-2, cyclooxygenase-2; TNC, tenascin-C; $S U V_{\text {max }}$, maximum of the standard uptake value; TLG, total lesion glycolysis; $S \mathrm{SV}_{\text {peak}}$, peak standard uptake value; Rad-score (PET), radiomics score derived from positron emission tomography imaging

\section{Prediction of LVSI in the multivariate logistic regression analysis}

In the multivariate logistic regression analysis, only the Rad-score and TNC were associated with LVSI in all patients with early-stage cervical cancer (Table 3).

Table 3

Multivariate logistic regression analysis

\begin{tabular}{|lllll|}
\hline Variable & Coefficient & SE & Wald & P \\
\hline TNC & 1.36497 & 0.45012 & 9.1958 & 0.0024 \\
\hline Rad-score (PET) & 1.20806 & 0.30471 & 15.7186 & 0.0001 \\
\hline Constant & -0.88808 & 0.38653 & 5.2789 & 0.0216 \\
\hline $\begin{array}{l}\text { SE, standard error; TNC, tenascin-C; Rad-score (PET), radiomics score derived from positron emission } \\
\text { tomography imaging. }\end{array}$ & & & \\
\hline
\end{tabular}

\section{Evaluation of the machine learning models}

The ROC curve was used to evaluate the efficiency of the machine learning models. In the training data set, three models all performed well in predicting LVSI (Table 4). The radiomics model had the best predictive performance among the models (AUC $=0.914 ; 95 \%$ confidence interval, $\mathrm{Cl}, 0.814-0.970 ; p<0.001$ ) in the training data set. The combined model had high sensitivity in predicting LVSI in the training data set (sensitivity $=1.0 ;$ specificity $=0.64 ; \mathrm{p}<0.001)($ Fig. 6).

However, in the testing data set, the combination of radiomics, COX-2, and TNC for predicting LVSI had better efficiency than the other models (AUCs: 0.801 vs. 0.756 and 0.801 vs. 0.631 , respectively; sensitivity $=0.67$; specificity $=1.00$ ). The performance of the protein model was not statistically significant (AUC = $0.631, p=0.238)$ (Fig. 7). 
Table 4

Performance of each model in the training and testing data sets

\begin{tabular}{|c|c|c|c|c|c|c|c|c|}
\hline \multirow[t]{2}{*}{ Model } & \multicolumn{4}{|c|}{ Training data set } & \multicolumn{4}{|c|}{ Testing data set } \\
\hline & $\operatorname{AUC}(95 \% \mathrm{Cl})$ & $\mathbf{p}$ & Sen & Spe & AUC (95\% Cl) & p & Sen & Spe \\
\hline $\begin{array}{l}\text { Radiomics } \\
\text { model }\end{array}$ & $\begin{array}{l}0.914(0.814- \\
0.970)\end{array}$ & $<.001$ & 0.93 & 0.74 & $\begin{array}{l}0.756(0.545- \\
0.904)\end{array}$ & 0.014 & 0.58 & 0.92 \\
\hline Protein model & $\begin{array}{l}0.756(0.630- \\
0.857)\end{array}$ & $<.001$ & 0.90 & 0.48 & $\begin{array}{l}0.631(0.417- \\
0.814)\end{array}$ & 0.238 & 0.50 & 0.77 \\
\hline $\begin{array}{l}\text { Combined } \\
\text { model }\end{array}$ & $\begin{array}{l}0.905(0.803- \\
0.965)\end{array}$ & $<.001$ & 1.00 & 0.64 & $\begin{array}{l}0.801(0.594- \\
0.932)\end{array}$ & 0.003 & 0.67 & 1.00 \\
\hline
\end{tabular}

\section{Pairwise comparison of the ROC curves of the models}

In the training data set, the AUC of the ROC of the combined model was better than that of the protein model. Furthermore, the radiomics model provided better prediction performance than the protein model (Table 5). However, in the testing dataset, the result of the DeLong test showed that the AUC of ROC curves of the three models were not significantly different (Table 6).

Table 5

DeLong test of ROC curve of models in the training data set

\begin{tabular}{|llll|}
\hline Pairwise comparison of ROC curves & Difference between areas & $\mathbf{Z}$ & $\mathbf{p}$ \\
\hline Radiomics and protein models & 0.158 & 2.196 & $0.028^{*}$ \\
\hline Radiomics and combined models & 0.008 & 0.619 & 0.536 \\
\hline Protein and combined models & 0.149 & 2.317 & $0.021^{*}$ \\
\hline *Statistically significant, $p<0.05$. & & & \\
\hline
\end{tabular}

ROC, receiver operating characteristics.

Table 6

DeLong test of ROC curve of models in the testing data set

\begin{tabular}{|llll|}
\hline Pairwise comparison of ROC curves & Difference between areas & $\mathbf{Z}$ & p \\
\hline Radiomics and protein models & 0.125 & 0.794 & 0.427 \\
\hline Radiomics and combined models & 0.045 & 1.265 & 0.206 \\
\hline Protein and combined models & 0.170 & 1.192 & 0.233 \\
\hline ROC, receiver operating characteristics. & & \\
\hline
\end{tabular}




\section{Discussion}

According to the 2018 FIGO criteria[15], surgery is the primary treatment for patients with early-stage squamous cervical carcinoma. LVSI has an important influence on surgery and patient prognosis according to the FIGO criteria[15]. Although most patients have excellent prognosis, approximately $30 \%$ patients might have recurrence and decreased survival rate[20]. Many studies have proven that LVSI is closely associated with prognosis and is an independent risk factor[2-4, 6, 7]. The expression of some proteins, including COX-2, TNC, and others, are related to LVSI, tumor microenvironment, and inflammation[11, 12, 21]. Hence, accurate and early assessment of LVSI are important in prognosis assessment and treatment decision making in order to ensure that patients can obtain the maximum treatment benefit.

Our study aimed to establish a machine learning model that combines radiomics derived from PET images with molecular proteins that are associated with the pathology of cervical cancer in order to predict LVSI in patients with early-stage cervical cancer. The results indicate that the Rad-score was closely correlated with LVSI, and there were statistically significant differences in COX-2, TNC, and the Rad-score between the LVSI and non-LVSI groups. Moreover, we determined that the model based on the Rad-score could predict LVSI. When the Rad-score and molecular protein expression were combined, the AUC of the model improved a bit in the testing data set, but the DeLong test showed no statically significant difference between the two models in the testing data set.

Malignant tumors exhibit intratumoral biological heterogeneity and lead to changes in the texture parameters of the corresponding primary tumor on PET images. A previous study determined that heterogenic FDG uptake within a tumor correlated with intratumoral histopathological appearance[22]. Mu et al[23] found that inverse difference moment and correlation showed statistically significant differences between the early (stages I and II) and advanced stages (stages III and IV) of cervical cancer. Similarly, inverse difference moment and correlation were selected to calculate the Rad-score for predicting LVSI in our study. Recently, Li et al[17] study showed that the PET textures of primary tumor could predict lymphatic metastasis in early-stage cervical carcinoma (AUC $=0.757$ in the validation data set; $95 \% \mathrm{Cl}$, $0.545-0.904 ; p<0.05)$. Other research have also shown that radiomics of primary tumor based on PET images could reflect tumor malignancy and were associated with nodal metastases and molecular subtypes of solid tumor[24, 25]. The Rad-score[26], which is calculated by the linear combination of selected features (including histogram and texture parameters) weighted by their respective coefficients selected as informative features, is usually used for radiomics analysis. In the present study, the MannWhitney $\mathrm{U}$ test showed that the Rad-score and TNC had significant differences between the LVSI and nonLVSI in all data sets, and the LVSI group had a higher Rad-score than the non-LVSI group in Fig. 5.

A previous research found that the molecular expression of some proteins was correlated with LVSI. Normal cervical tissues have weak expression of TNC and COX-2. Previously, Pilch et al[13] determined that, in invasive cervical carcinoma, TNC expression was markedly increased. Other studies have proven that TNC has a significant role in tumor growth, migration, metastasis, angiogenesis, and stromal inflammation[14, 27, 28]. The study of Liu et al[11] found that COX-2 expression was associated with 
lymphangiogenesis and lymph node metastasis in cervical cancer. Similarly, Hoellen et al[12] proved that COX-2 expression was significantly associated with LVSI $(p=0.017)$. Similarly, our study also found that the differences in COX-2 and TNC between the LVSI and non-LVSI groups were statically significant. In the multivariate logistic regression, TNC expression was associated with LVSI. Furthermore, COX-2 had a slight correlation with TNC (Fig. 4). We hypothesized that the result may be caused by the inflammatory microenvironment of the tumor. Liu et al[11] demonstrated that COX-2 may promote cancer progression and metastasis by enhancing the expression of vascular endothelial growth factor $\mathrm{C}$ and other mechanisms. The research showed that TNC could also facilitate the formation of cancer stroma, including desmoplasia and angiogenesis, and enhanced inflammation in the cancer stroma may augment macrophage recruitment and secretion of tumor-promoting and inflammatory cytokines by macrophages and fibroblasts[14]. Thus, TNC and COX-2 were selected to predict LVSI in cervical cancer.

Although some protein expression and radiomics were associated with LVSI in cervical carcinoma, the correlation of molecular proteins and radiomics has been rarely explored and confirmed. In our study, the Rad-score was correlated with TNC, according to the Spearman correlation analysis (Fig. 4). Thus, we assumed that the PET imaging textures of primary tumors changed through TNC and other proteins. The textures of primary tumor reflect the heterogeneity of tumor, and they could be used to predict LVSI in cervical carcinoma. Another research also hoped to utilize the expression of TNC on PET imaging by devising a new PET tracer[29]. However, our result initially showed that radiomics derived from PET imaging provided a new possibility for non-invasive visualization of TNC expression. Song et al[30] also found that the image signal changes on magnetic resonance imaging (MRI) were consistent with TNC expression, and cervical cancer tissues with node metastasis had the highest TNC expression.

Three machine learning models were established with logistic regression algorithm in the training data set and evaluated in the testing data set. All three models performed well in the training data set (Table 4), but the radiomics model had the highest AUC in the training data set. However, in the testing data set, the AUC value of the combined model was higher than that of the other models (Table 4). The reason for the results was that our data set was slightly smaller. Thus, we used all data sets to perform the multivariate logistic regression analysis (Table 3). The results also showed that the Rad-score and TNC were associated with LVSI in all data sets. Two different methods (statistics and machine learning) both confirmed that the combination of radiomics and TNC could predict LVSI in early-stage cervical cancer. The combined model for predicting LVSI was credible. The DeLong test also indicated that the AUC of the ROC of the combined model was better than that of the protein model in the training data set (Table 5). Previously, a few researchers also wanted to predict or distinguish LVSI through radiology for cervical cancer. Yang et al[31] determined found that the minimum apparent diffusion coefficient and the minimum apparent diffusion coefficient ratio were significantly lower in LVI-positive invasive cervical cancer than in LVI-negative invasive cervical cancer $\left(0.772 \pm 0.062\right.$ vs. $0.917 \pm 0.052, p<0.001$, and $0.712 \pm 0.078 \times 10^{-3}$ vs. $0.867 \pm$ $0.099 \times 10^{-3} \mathrm{~mm}^{2} / \mathrm{s}, \mathrm{p}<0.001$, respectively). Gross tumor volume[32] on MRI was also identified to be a possible independent risk factor for predicting LVSI (AUC $=0.700, p<0.05$ ). Recently, the use of radiomics based on magnetic resonance for predicting LVSI has been studied. According to Hua et al[33], the model based on multiparametric MRI showed the best prediction results, with an AUC of $0.842(95 \% \mathrm{Cl}, 0.772-$

Page $12 / 23$ 
0.913 ; sensitivity $=0.773$; specificity $=0.776)$ in the training cohort and $0.775(95 \% \mathrm{Cl}, 0.637-0.912$; sensitivity $=0.739$; specificity $=0.667)$ in the validation cohort. Similarly, Li et al[34] also found that the radiomics nomogram derived from MRI showed favorable discrimination between LVSI and non-LVSI groups, with an AUC of $0.754(95 \% \mathrm{Cl}, 0.6326-0.8745)$ in the training cohort and $0.727(95 \% \mathrm{Cl}, 0.5449-$ 0.9097) in the validation cohort. We initially used the combination of PET radiomics with protein molecule to predict LVSI, which showed that the radiomics and combined models based on ${ }^{18} \mathrm{~F}-\mathrm{FDG}$ PET imaging showed better results than those of previous studies.

However, this study has some limitations. First, the size of the data set was inadequate; thus, we need a larger number of data set to test our models as well as multicenter imaging data to evaluate reproducibility. Second, we only analyzed the association of the expression of TNC and COX-2 with LVSI. In the future, we hope to perform more protein analyses and explore the correlation of DNA with LVSI and the function of radiogenomics in order to predict LVSI. Finally, the PET image resolution was low, thus limiting the precision of the segment of tumor $\mathrm{VOI}$ as well as the extraction of the radiomics features.

\section{Conclusion}

The results of this study indicated that LVSI in patients with early-stage cervical cancer can be predicted by radiomics derived from PET imaging. The machine learning model that combines PET-based radiomics with COX-2 and TNC provides a new tool for detecting LVSI. The combined model improved prediction accuracy. In addition, the Rad-score derived from PET image textures is associated with TNC expression.

\section{Abbreviations}

Lymphovascular space invasion (LVSI), cyclooxygenase-2 (COX2), tenascin-C (TNC), International Federation of Gynecology and Obstetrics (FIGO), magnetic resonance imaging (MRI), area under ROC curves (AUC), Receiver operating characteristic (ROC), radiomics score (Rad-score), least absolute shrinkage and selection operator (LASSO)

\section{Declarations}

\section{Funding}

This study was funded by LIAONING Science \& Technology Project (2017225012), LIAONING Science Natural Science Foundation (2019-MS-373) and 345 Talent Project.

\section{Conflict of Interest}

The authors declare that they have no conflict of interest.

\section{Ethics approval}


This is a retrospective observational study. Our hospital Ethics Committee has confirmed that no ethical approval is required.

\section{Informed consent}

For this type of retrospective study in PET imaging, formal consent was not required.

\section{Availability of data and materials}

The datasets used and analyzed of the current study are available from the corresponding author on reasonable request.

\section{Consent for publication}

Publication is approved by all authors and tacitly or explicitly by the responsible authorities where the work was carried out.

\section{Authors' contributions}

Conceptualization, Xiaoran Li, Chen Xu; Methodology, Xiaoran Li, Yan Guo ; Formal Analysis, Xiaoran Li; Investigation, Xiaoran Li; Resources, Xiaoran Li; Data Curation, Yang Yu, Chen Xu; Writing-Original Draft Preparation, Xiaoran Li; Writing - Review \& Editing, Xiaoran Li; Supervision, Hongzan Sun; Project Administration, Hongzan Sun; Funding Acquisition, Hongzan Sun. All authors read and approved the final manuscript.

\section{Acknowledgements}

We would like to thank the native English-speaking scientists of BioMed Proofreading Company for editing our manuscript.

\section{References}

1. Torre LA, Bray F, Siegel RL, Ferlay J, Lortet-Tieulent J, Jemal A. Global cancer statistics, 2012. Cancer J Clin. 2015;65(2):87-108.

2. Delgado G. Lymphovascular space involvement in cervical cancer: an independent risk factor. Gynecol Oncol. 1998;68(3):219.

3. Herr D, Konig J, Heilmann V, Koretz K, Kreienberg R, Kurzeder C. Prognostic impact of satellitelymphovascular space involvement in early-stage cervical cancer. Ann Surg Oncol. 2009;16(1):12832.

4. Memarzadeh S, Natarajan S, Dandade DP, Ostrzega N, Saber PA, Busuttil A, Lentz SE, Berek JS. Lymphovascular and perineural invasion in the parametria: a prognostic factor for early-stage cervical cancer. Obstetrics gynecology. 2003;102(3):612-9. 
5. Pol FJ, Zusterzeel PL, van Ham MA, Kuijpers DA, Bulten J, Massuger LF. Satellite lymphovascular space invasion: An independent risk factor in early stage cervical cancer. Gynecol Oncol. 2015;138(3):579-84.

6. Yan W, Qiu S, Ding Y, Zhang Q, Si L, Lv S, Liu L. Prognostic value of lymphovascular space invasion in patients with early stage cervical cancer in Jilin, China: A retrospective study. Medicine. 2019;98(40):e17301.

7. Magno V, Rivoire WA, Monego H, Appel M, dos Reis R, Hammes LS, Edelweiss MI, Capp E. Prevalence and Relapse Impact of Lymphovascular Invasion at Early-Stage Cervical Cancer: Prevalence of Lymphovascular Invasion in Cervical Cancer. J Low Genit Tract Di. 2009;13(4):256-9.

8. Robert J. Gillies PEK, Hedvig Hricak. Radiomics: Images Are More than Pictures, They Are Data. Radiology. 2016;278(2):563-77.

9. Schernberg A, Reuze S, Orlhac F, Buvat I, Dercle L, Sun R, Limkin E, Escande A, Haie-Meder C, Deutsch E, et al: A score combining baseline neutrophilia and primary tumor SUVpeak measured from FDG PET is associated with outcome in locally advanced cervical cancer. European journal of nuclear medicine and molecular imaging 2017.

10. Lambin P, Rios-Velazquez E, Leijenaar R, Carvalho S, van Stiphout RG, Granton P, Zegers CM, Gillies R, Boellard R, Dekker A, et al. Radiomics: extracting more information from medical images using advanced feature analysis. European journal of cancer. 2012;48(4):441-6.

11. Liu H, Xiao J, Yang Y, Liu Y, Ma R, Li Y, Deng F, Zhang Y. COX-2 expression is correlated with VEGF-C, lymphangiogenesis and lymph node metastasis in human cervical cancer. Microvasc Res. 2011;82(2):131-40.

12. Hoellen F, Waldmann A, Banz-Jansen C, Rody A, Heide M, Koster F, Ribbat-Idel J, Thorns C, Gebhard M, Oberlander $\mathrm{M}$, et al. Expression of cyclooxygenase-2 in cervical cancer is associated with lymphovascular invasion. Oncology letters. 2016;12(4):2351-6.

13. Pilch HS, Schlenger U, Lautz K, Tanner A, Hoeckel B, Knapstein M. P. G.: Expression of Tenascin in Human Cervical Cancer-Association of Tenascin Expression with Clinicopathological Parameters. Gynecol Oncol. 1999;73(3):415-21.

14. Yoshida T, Akatsuka T, Imanaka-Yoshida K. Tenascin-C and integrins in cancer. Cell adhesion migration. 2015;9(1-2):96-104.

15. Bhatla N, Aoki D, Sharma DN, Sankaranarayanan R. Cancer of the cervix uteri. Int J Gynaecol Obstet. 2018;143(Suppl 2):22-36.

16. Yeo W, Chan SL, Mo FK, Chu CM, Hui JW, Tong JH, Chan AW, Koh J, Hui EP, Loong H, et al. Phase I/II study of temsirolimus for patients with unresectable Hepatocellular Carcinoma (HCC)- a correlative study to explore potential biomarkers for response. BMC Cancer. 2015;15:395.

17. Li K, Sun H, Lu Z, Xin J, Zhang L, Guo Y, Guo Q. Value of [(18)F]FDG PET radiomic features and VEGF expression in predicting pelvic lymphatic metastasis and their potential relationship in early-stage cervical squamous cell carcinoma. Eur J Radiol. 2018;106:160-6. 
18. Parmar C, Barry JD, Hosny A, Quackenbush J, Aerts H. Data Analysis Strategies in Medical Imaging. Clinical cancer research: an official journal of the American Association for Cancer Research. 2018;24(15):3492-9.

19. Tibshirani R. The lasso method for variable selection in the Cox model. Statistics in medicine. 1997;16(4):385-95.

20. Ahn HK, Shin JW, Ahn HY, Park CY, Lee NW, Lee JK, Hwang IC. Metabolic components and recurrence in early-stage cervical cancer. Tumour biology: the journal of the International Society for Oncodevelopmental Biology Medicine. 2015;36(3):2201-7.

21. Pollanen RS, Vuopala Y, Laara S, Lehto E. V P: Tenascin in human papillomavirus associated lesions of the uterine cervix. J Clin Pathol. 1996;49(6):521-3.

22. Henriksson EKE, Wahlberg P, Ohlsson T, Wennerberg J, Brun E. 2-Deoxy-2-[18F] fluoro-D-glucose uptake and correlation to intratumoral heterogeneity. ANTICANCER RESEARCH. 2007;27(4B):2155-9.

23. Mu W, Chen Z, Liang Y, Shen W, Yang F, Dai R, Wu N, Tian J. Staging of cervical cancer based on tumor heterogeneity characterized by texture features on (18)F-FDG PET images. Physics in medicine biology. 2015;60(13):5123-39.

24. De Bernardi E, Buda A, Guerra L, Vicini D, Elisei F, Landoni C, Fruscio R, Messa C, Crivellaro C. Radiomics of the primary tumour as a tool to improve (18)F-FDG-PET sensitivity in detecting nodal metastases in endometrial cancer. EJNMMI research. 2018;8(1):86.

25. Yin Q, Hung SC, Rathmell WK, Shen L, Wang L, Lin W, Fielding JR, Khandani AH, Woods ME, Milowsky $\mathrm{MI}$, et al. Integrative radiomics expression predicts molecular subtypes of primary clear cell renal cell carcinoma. Clinical radiology. 2018;73(9):782-91.

26. Yanqi Huang M, Zaiyi Liu M, Lan He M, Xin Chen M, Dan Pan M, Zelan Ma M, Cuishan Liang M, Jie Tian P, Changhong Liang M. Radiomics Signature: A Potential Biomarker for the Prediction of DiseaseFree Survival in Early-Stage (I or II) Non-Small Cell Lung Cancer. Radiology. 2016;281(3):947-57.

27. Xia S, Lal B, Tung B, Wang S, Goodwin CR, Laterra J. Tumor microenvironment tenascin-C promotes glioblastoma invasion and negatively regulates tumor proliferation. Neurooncology. 2016;18(4):50717.

28. Hancox RA, Allen MD, Holliday DL, Edwards DR, Pennington CJ, Guttery DS, Shaw JA, Walker RA, Pringle $\mathrm{JH}$, Jones JL. Tumour-associated tenascin-C isoforms promote breast cancer cell invasion and growth by matrix metalloproteinase-dependent and independent mechanisms. Breast cancer research: BCR. 2009;11(2):R24.

29. Jacobson O, Yan X, Niu G, Weiss ID, Ma Y, Szajek LP, Shen B, Kiesewetter DO, Chen X. PET imaging of tenascin-C with a radiolabeled single-stranded DNA aptamer. Journal of nuclear medicine: official publication Society of Nuclear Medicine. 2015;56(4):616-21.

30. Song J, Hu Q, Huang J, Chen T, Ma Z, Shi H. MR targeted imaging for the expression of tenascin-C in cervical cancer. Br J Radiol. 2018;91(1090):20170681.

31. Yang W, Qiang JW, Tian HP, Chen B, Wang AJ, Zhao JG. Minimum apparent diffusion coefficient for predicting lymphovascular invasion in invasive cervical cancer. Journal of magnetic resonance 
imaging: JMRI. 2017;45(6):1771-9.

32. Chen XL, Chen GW, Xu GH, Ren J, Li ZL, Pu H, Li H. Tumor Size at Magnetic Resonance Imaging Association With Lymph Node Metastasis and Lymphovascular Space Invasion in Resectable Cervical Cancer: A Multicenter Evaluation of Surgical Specimens. International journal of gynecological cancer: official journal of the International Gynecological Cancer Society. 2018;28(8):1545-52.

33. Hua W, Xiao T, Jiang X, Liu Z, Wang M, Zheng H, Wang S. Lymph-vascular space invasion prediction in cervical cancer: Exploring radiomics and deep learning multilevel features of tumor and peritumor tissue on multiparametric MRI. Biomed Signal Process Control. 2020;58:101869.

34. Li Z, Li H, Wang S, Dong D, Yin F, Chen A, Wang S, Zhao G, Fang M, Tian J, et al: MR-Based Radiomics Nomogram of Cervical Cancer in Prediction of the Lymph-Vascular Space Invasion preoperatively. Journal of magnetic resonance imaging: JMRI 2018.

\section{Figures}

\section{Features $(n=401)$}
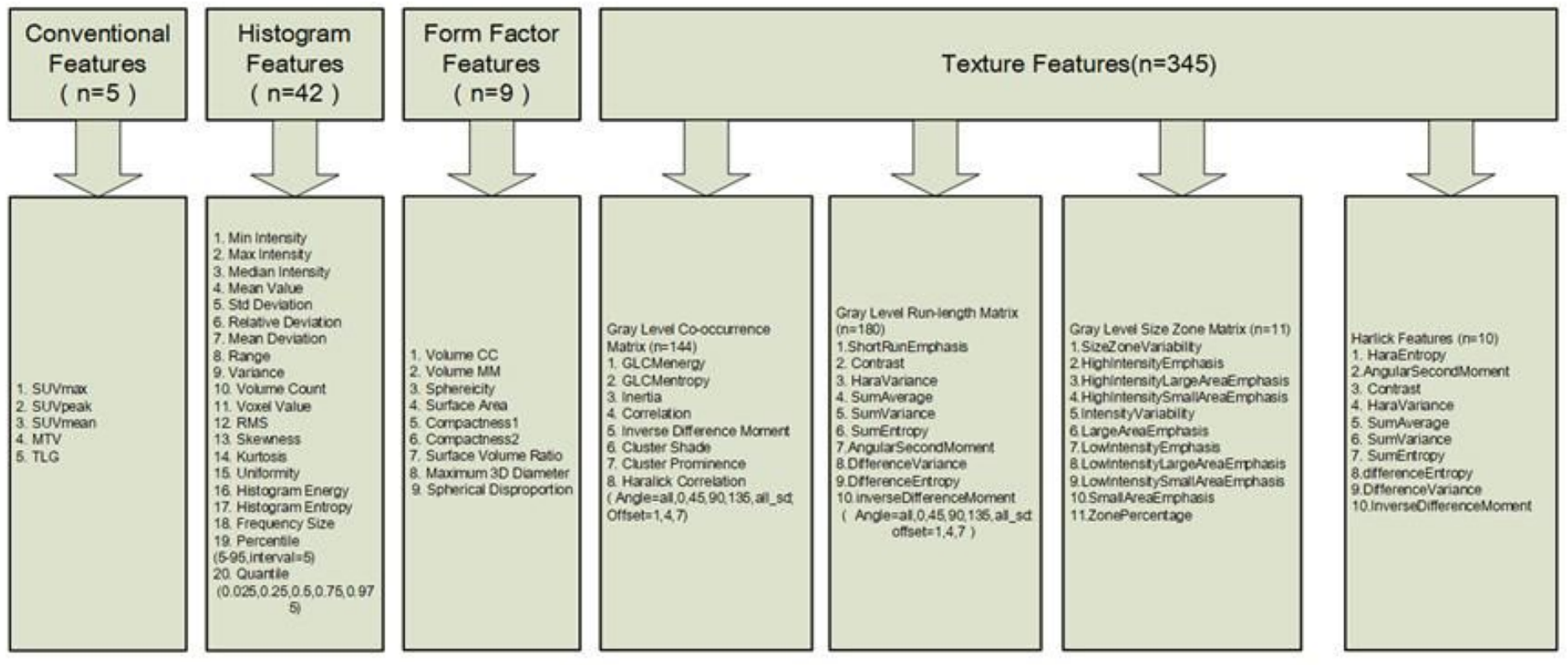

Figure 1 
A total of 401 features were extracted from PET images, which consist of conventional features $(n=5)$, and 396 radiomics features including histogram features $(n=42)$, form factor features $(n=9)$ and texture features $(n=345)$.
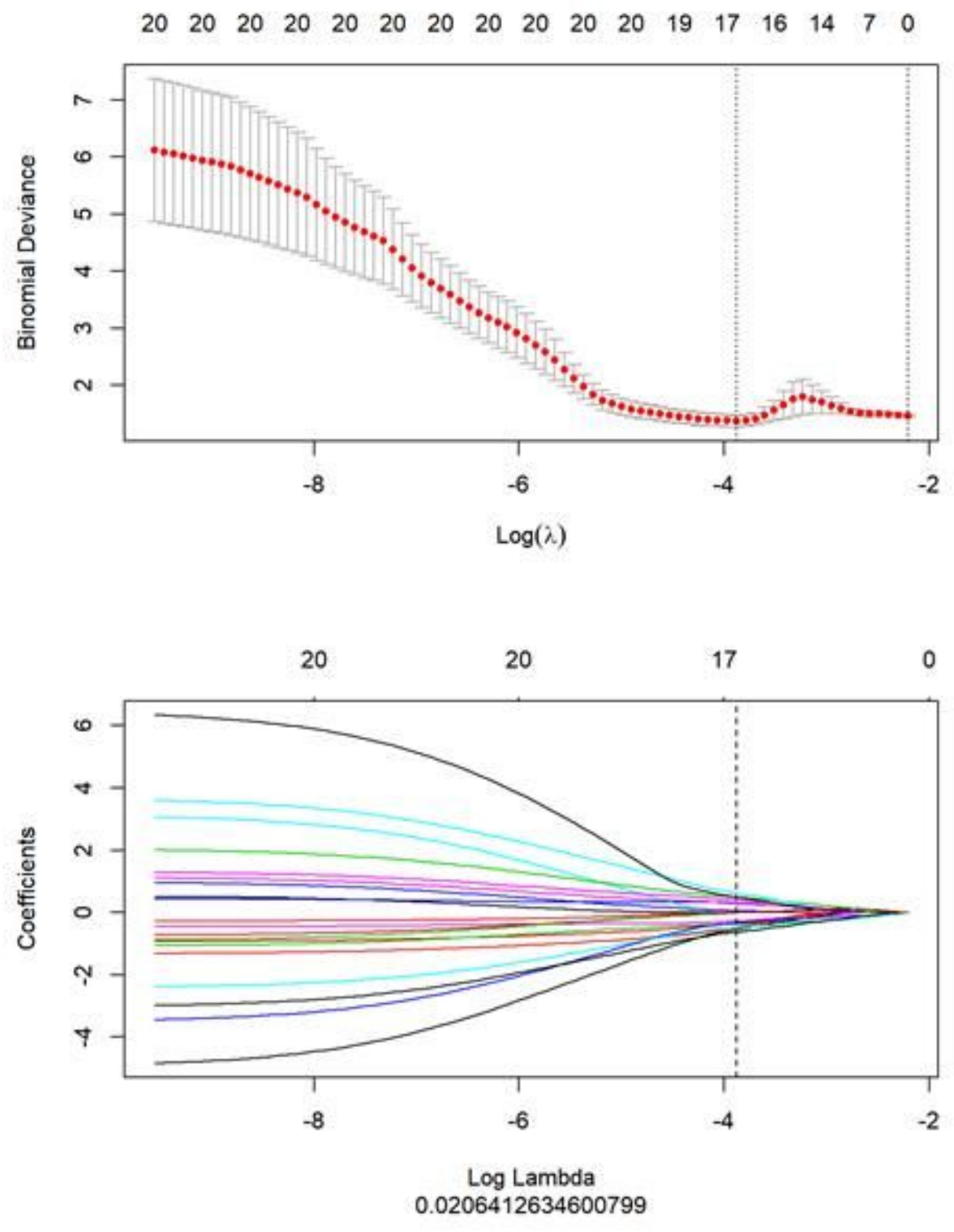

Figure 2

The least absolute shrinkage and selection operator was used to select informative features. When lambda was 0.02 in the 10 -fold cross validation, the binomial deviance had the minimum number. 


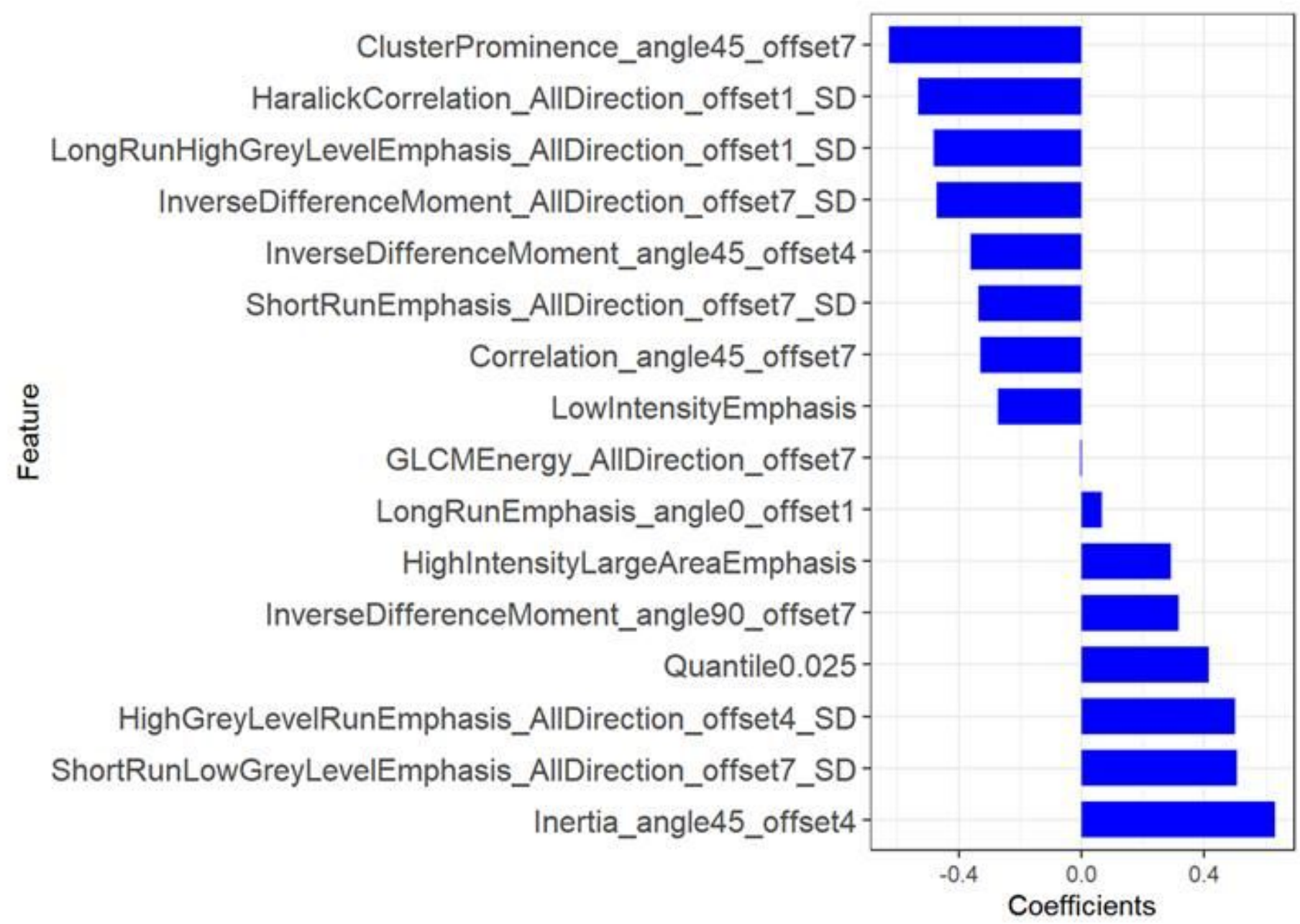

\section{Figure 3}

Sixteen most informative features reserved for the Rad-score calculation after least absolute shrinkage and selection operator selection. The abscissa is the size of the correlation coefficient. The ordinate represents the feature names. 


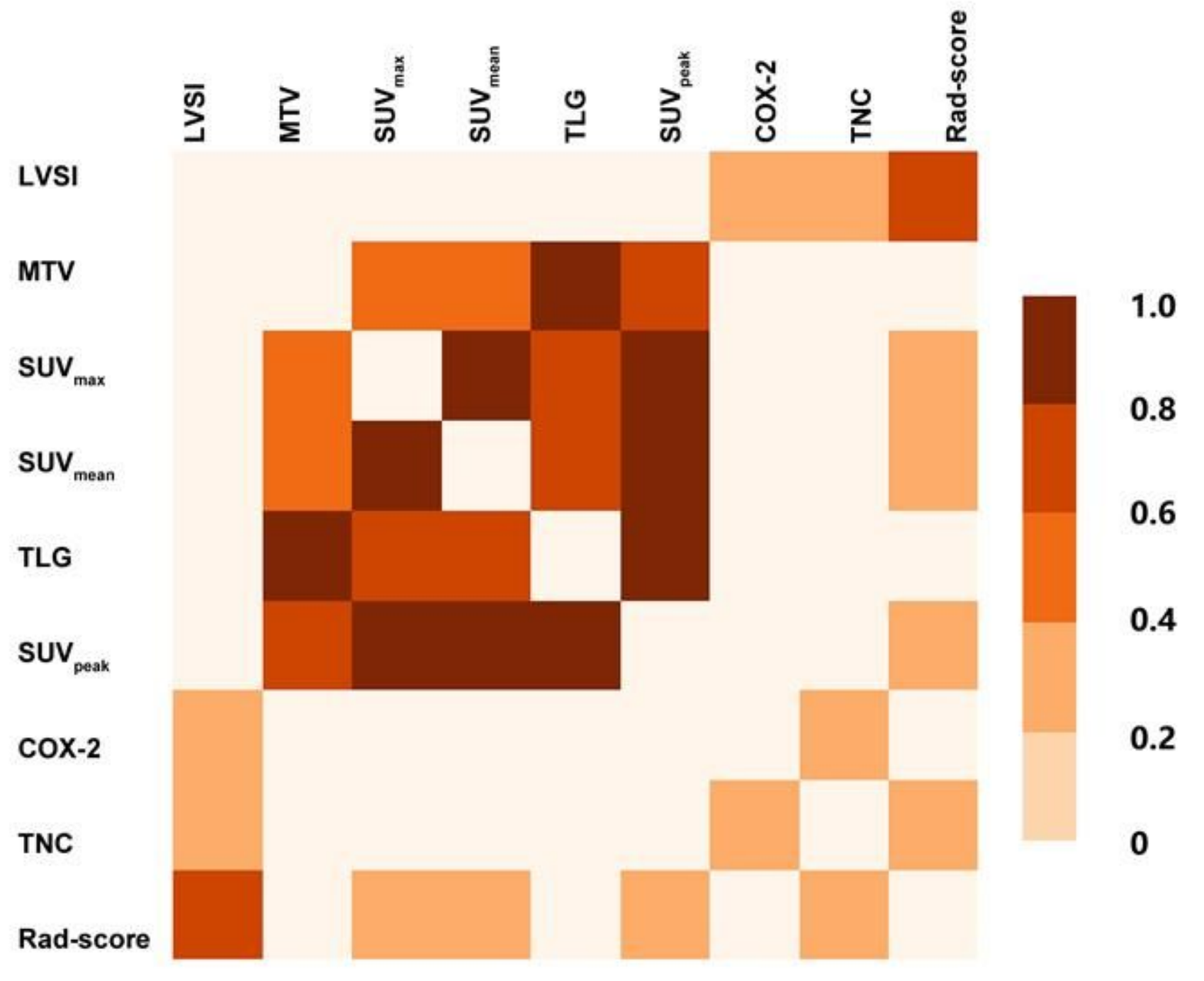

\section{Figure 4}

Heatmap of the correlation of LVSI, Rad-score, conventional parameters, TNC, and COX-2. The color scale on the right represents the interval of statistically significant regression coefficients. White squares indicate no statistical significance ( $p>0.05)$. LVSI, lymphovascular space invasion; MTV, metabolic tumor volume; SUVmax, maximum of the standard uptake value; SUVmean, mean standard uptake value; TLG, total lesion glycolysis; SUVpeak, peak standard uptake value; COX-2, cyclooxygenase-2; TNC, tenascin-C; Rad-score, radiomics score. 


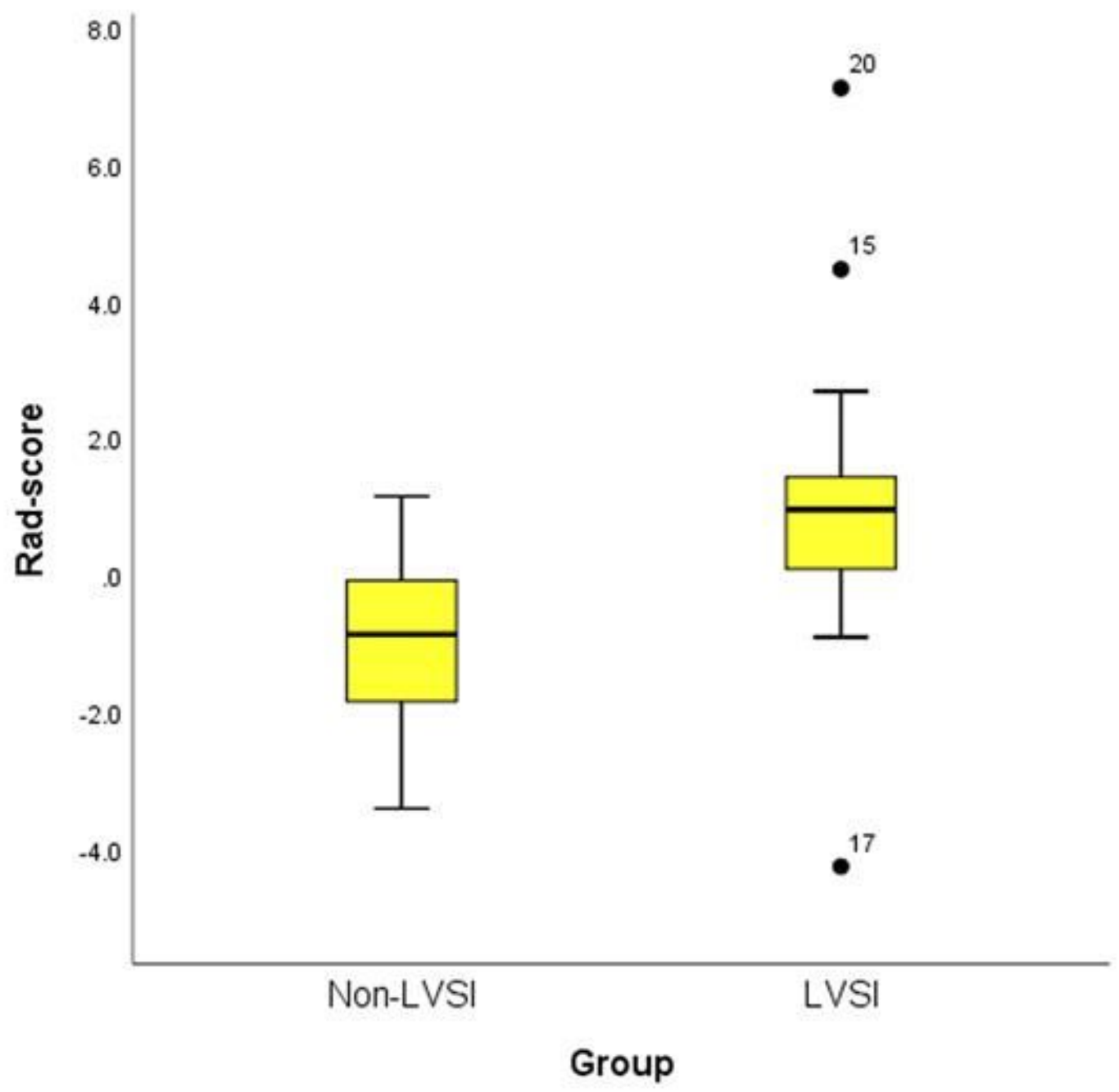

Figure 5

The Rad-score of patients with LVSI was significantly higher than those without LVSI. Rad-score (PET), radiomics score derived from positron emission tomography imaging. 


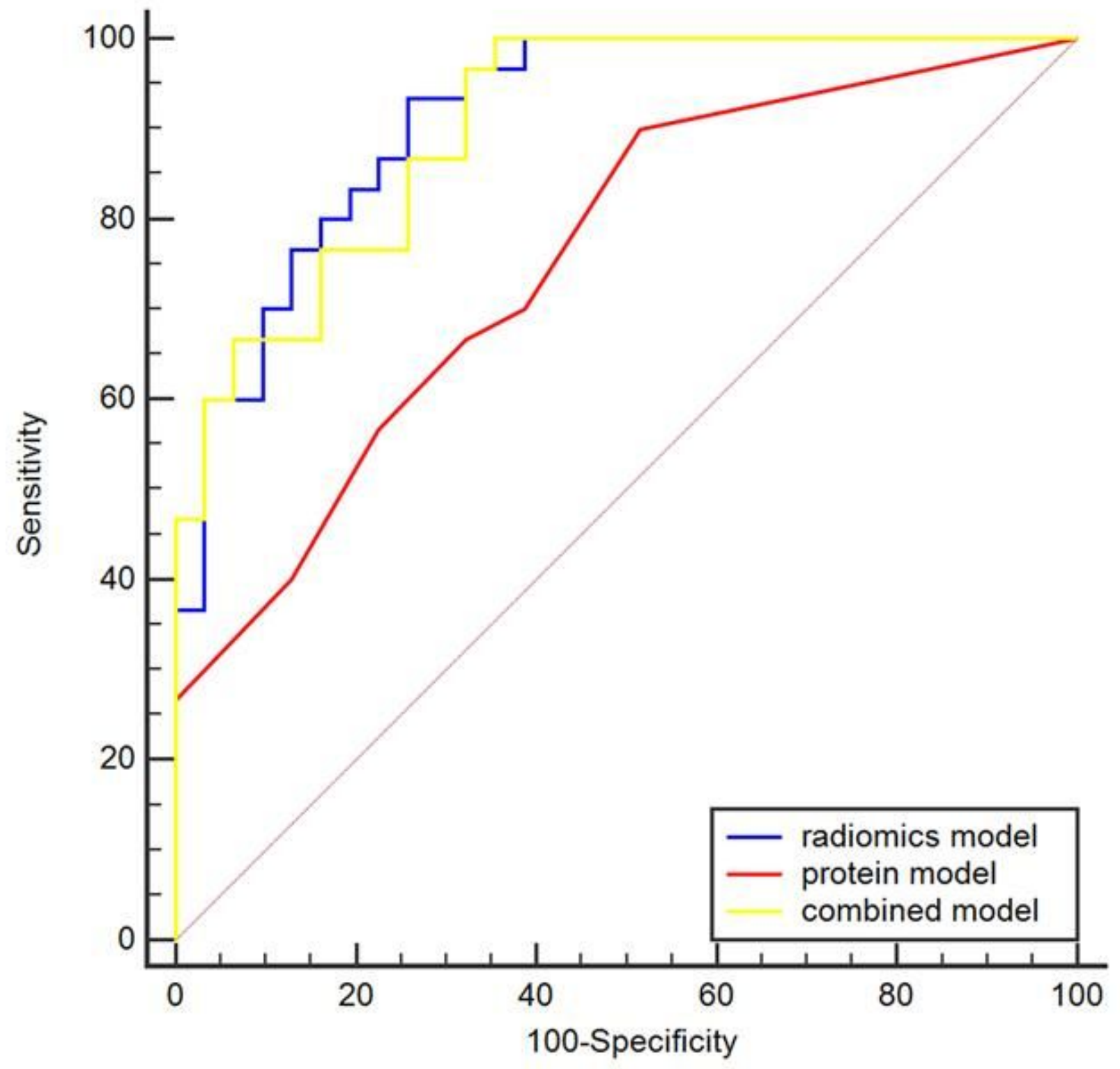

Figure 6

Receiver operating characteristics curves of the radiomics, proteins, and combined models in the training data set. Blue curve, radiomics model; red curve, protein model; yellow curve, combined model. 


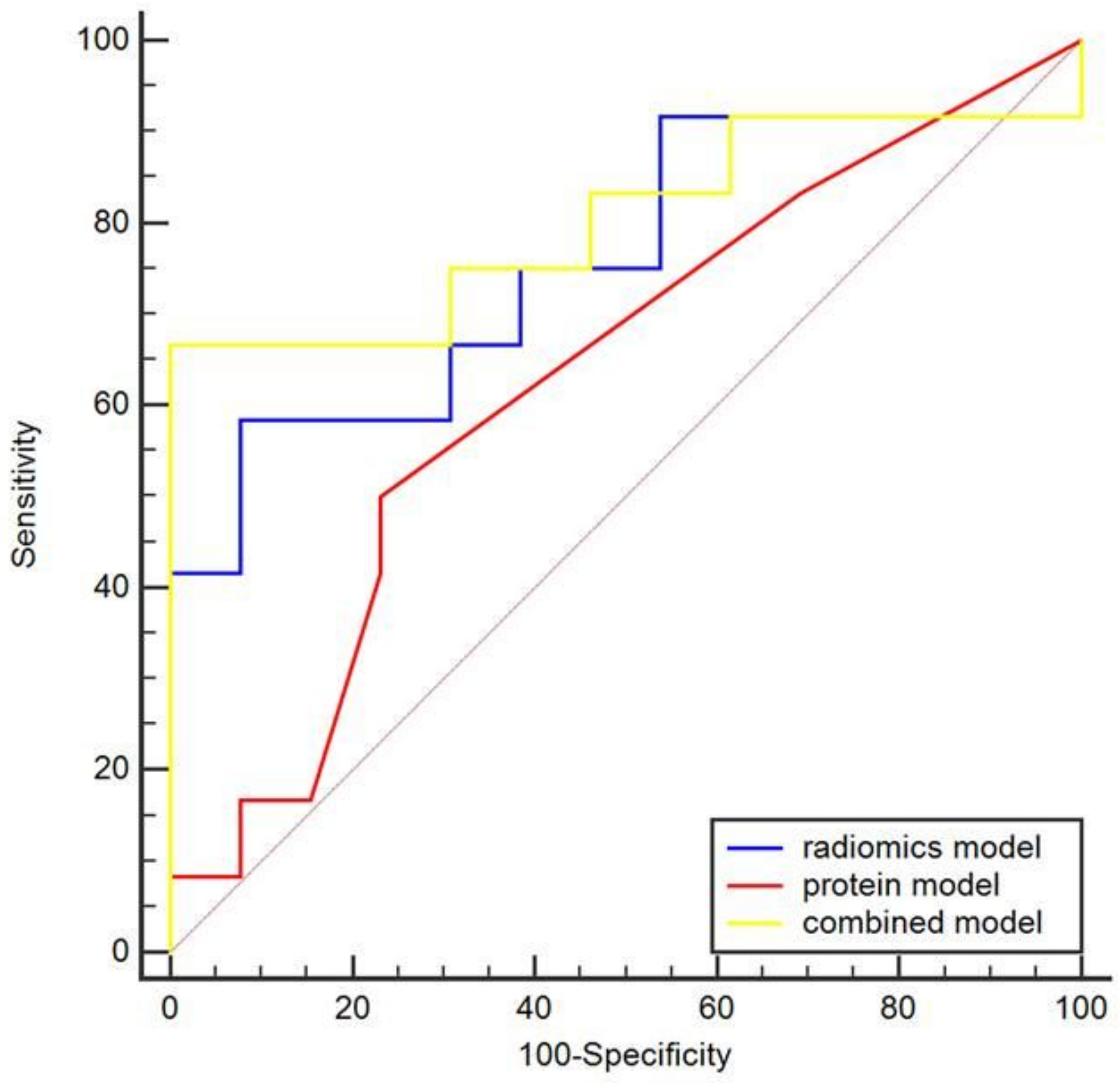

Figure 7

Receiver operating characteristics curves of the radiomics, protein, and combined models in the testing data set. Blue curve, radiomics model; red curve, protein model; yellow curve, combined model. 